ARTICLE

Received 24 Jul 2013 | Accepted 12 Nov 2013 | Published 13 Dec 2013 DOl: 10.1038/ncomms3916

\title{
UTX coordinates steroid hormone-mediated autophagy and cell death
}

Donna Denton 1,2, May T. Aung-Htut ${ }^{1}$, Nirmal Lorensuhewa', Shannon Nicolson', Wenying Zhu', Kathryn Mills', Dimitrios Cakouros ${ }^{1}$, Andreas Bergmann ${ }^{3} \&$ Sharad Kumar ${ }^{1,2,4}$

Correct spatial and temporal induction of numerous cell type-specific genes during development requires regulated removal of the repressive histone $\mathrm{H} 3$ lysine 27 trimethylation (H3K27me3) modification. Here we show that the H3K27me3 demethylase dUTX is required for hormone-mediated transcriptional regulation of apoptosis and autophagy genes during ecdysone-regulated programmed cell death of Drosophila salivary glands. We demonstrate that dUTX binds to the nuclear hormone receptor complex Ecdysone Receptor/Ultraspiracle, and is recruited to the promoters of key apoptosis and autophagy genes. Salivary gland cell death is delayed in DUTX mutants, with reduced caspase activity and autophagy that coincides with decreased apoptosis and autophagy gene transcripts. We further show that salivary gland degradation requires dUTX catalytic activity. Our findings provide evidence for an unanticipated role for UTX demethylase activity in regulating hormone-dependent cell death and demonstrate how a single transcriptional regulator can modulate a specific complex functional outcome during animal development.

\footnotetext{
${ }^{1}$ Centre for Cancer Biology, SA Pathology, Frome Road, Adelaide, South Australia 5000, Australia. ${ }^{2}$ Division of Health Sciences, University of South Australia, Adelaide, South Australia 5001, Australia. ${ }^{3}$ Department of Cancer Biology, University of Massachusetts Medical School, Worcester, Massachusetts 01605 , USA. ${ }^{4}$ Department of Medicine, The University of Adelaide, Adelaide, South Australia 5005, Australia. Correspondence and requests for materials should be addressed to S.K. (email: Sharad.Kumar@health.sa.gov.au).
} 
P rogrammed cell death (PCD) is essential for animal development and is required for tissue modelling, deleting harmful cells and to maintain homeostasis. In response to various signals such as cytotoxic insults, hormones and growth factors the control of the transcriptional balance between prosurvival and pro-death genes can modulate PCD. Nuclear hormone receptors (NR) play essential roles in spatial and temporal regulation of gene transcription as they recruit histonemodifying enzymes to gene promoters/enhancers ${ }^{1,2}$. These multiprotein complexes modulate the expression of distinct gene networks regulating diverse biological processes such as cell proliferation, differentiation and $\mathrm{PCD}^{1-3}$.

An important determinant of the transcriptional status of a gene is the histone methylation profile. The methylation of lysine residues on histones is site-specific and highly regulated by histone lysine methyltransferases (KMT) and lysine demethylases $(\mathrm{KDM})^{4,5}$. Generally, methylation of histone 3 on lysine 4 (H3K4), lysine $36(\mathrm{H} 3 \mathrm{~K} 36)$ and lysine $79(\mathrm{H} 3 \mathrm{~K} 79)$ is associated with active transcription, whereas methylation of lysine 9 (H3K9) and lysine 27 (H3K27) is associated with repression. Depending on the context, histone methylation patterns may be stably maintained or amenable to change.

The highly conserved Polycomb group (PcG) and Trithorax group (TrxG) complexes are critical regulators of numerous developmental genes that act antagonistically in the maintenance of transcriptional repression or activation, respectively ${ }^{6}$. The PcG protein EZH2 is a KMT that trimethylates $\mathrm{H} 3 \mathrm{~K} 27$ facilitating gene repression. For gene expression, $\mathrm{H} 3 \mathrm{~K} 27 \mathrm{me} 3$ is removed and promoters acquire $\mathrm{H} 3 \mathrm{~K} 4 \mathrm{me} 3$ mediated by $\mathrm{TrxG}$ complexes ${ }^{7,8}$. The Jumonji (JMJ) domain-containing histone demethylases (HDMs) JMJD3 and UTX antagonize PcG-mediated silencing by removing the repressive methylation mark from H3K27me3, leading to an active chromatin state $e^{9-14}$. Recent studies have uncovered several roles for UTX including differentiation of mesoderm, embryonic stem cells and haematopoietic cells, cardiac development and myogenesis by remodelling chromatin, and facilitating the recruitment of appropriate transcriptional factors ${ }^{15-18}$. In addition to the demethylase functions, UTX appears to have enzyme-independent functions. While Utx is essential for murine embryonic viability and development, this is independent of demethylase function as Uty, which lacks demethylase activity, is able to compensate for Utx ${ }^{19-21}$. In addition, UTX associates with TrxG KMT (MLL2/3/ 4 ) and has demethylase-independent functions ${ }^{22,23}$. However, the roles of UTX in these demethylase-independent functions remain controversial.

The association of UTX with MLL and the importance of MLL complexes in NR-mediated gene regulation suggest that UTX may play a role in hormone-dependent transcriptional control. Indeed, several KDMs act as coregulators with the androgen receptor; however, a role for UTX in androgen receptor-mediated transcription has not yet been identified ${ }^{8}$. Despite the growing evidence for the role of $\mathrm{H} 3 \mathrm{~K} 27$ methylation in transcriptional regulation in stem cells, cell proliferation and cancer, the understanding of its role in hormone-dependent transcription and PCD is limited.

Nuclear receptor signaling pathways are highly conserved and in Drosophila the steroid hormone, 20-hydroxyecdysone (ecdysone), forms a complex with its heterodimeric nuclear hormone receptor, Ecdysone Receptor/Ultraspiracle (EcR/Usp), to modulate the transcription of many genes during major developmental transitions ${ }^{1,24}$. Following the larval-pupal transition a rise in ecdysone triggers the PCD of larval tissues no longer needed including the salivary glands ${ }^{25}$. The removal of the salivary glands depends on both apoptosis and autophagy and when both pathways are inhibited salivary gland PCD is blocked ${ }^{26}$. The expression of both apoptosis and autophagy genes is increased in dying salivary glands ${ }^{26-30}$. The transcriptional upregulation of the apoptosis initiator reaper $(r p r)$, caspase genes dronc and drice, and the adaptor dark in response to ecdysone is in part due to the direct binding of EcR/Usp to these promoters recruiting histonemodifying enzymes to activate apoptotic genes in specific tissues $31-36$.

Here we show that the Drosophila H3K27 demethylase orthologue, dUTX, drives temporal regulation of apoptosis and autophagy genes during ecdysone-mediated PCD of the salivary glands. Our data suggest that a physical association between dUTX and EcR/Usp results in their recruitment to promoters of key cell death and autophagy genes leading to H3K27me3 demethylation and subsequent gene expression during ecdysonemediated PCD.

\section{Results}

dUTX interacts with EcR and regulates ecdysone-induced PCD. Active gene transcription is generally associated with $\mathrm{H} 3 \mathrm{~K} 4$ trimethylation and removal of $\mathrm{H} 3 \mathrm{~K} 9$ and $\mathrm{H} 3 \mathrm{~K} 27$ trimethylation. We have found that in response to the hormone ecdysone, H3 associated with the promoters of cell death genes dronc, which encodes the essential apoptotic caspase in Drosophila and dark, which encodes the adaptor protein required for Dronc activation, acquires $\mathrm{H} 3 \mathrm{~K} 4 \mathrm{me} 3$ while losing $\mathrm{H} 3 \mathrm{~K} 9 \mathrm{me} 3$ (ref. 36). This suggests that HDMs are recruited to the promoters of key cell death genes to remove repressive histone marks such as $\mathrm{H} 3 \mathrm{~K} 9 \mathrm{me} 3$ and H3K27me3 during hormone-mediated transcription.

To determine which of the Jumonji HDM family members in Drosophila regulate PCD, we undertook a cell-based RNAi screen using cell lines that undergo ecdysone-mediated cell death ${ }^{37}$. Among the genes tested, we found that $d U T X$ knockdown resulted in significantly reduced ecdysone-mediated cell death and caspase activity (Fig. 1). As dUTX encodes a H3K27me3 demethylase, this suggested that the removal of $\mathrm{H} 3 \mathrm{~K} 27 \mathrm{me} 3$ may be important in hormone-mediated transcriptional control of PCD.

The temporal expression of the key apoptotic caspases dronc and drice during hormone-mediated PCD requires the binding of EcR/Usp directly to their promoter to enable the recruitment of coactivators ${ }^{33-35,38}$. Nuclear receptor coactivators frequently contain a LXXLL or LLXXL nuclear-receptor-interacting motif and sequence analysis of dUTX identified a potential motif LLQTL (amino acids 1,033-1,037 in isoform dUTX-RA) in the C-terminal region, which is conserved in mammalian UTX (LLRTL) $^{39}$. To determine whether the role of dUTX in cell death was through an interaction with EcR/Usp, we examined the interaction between the C-terminal region of dUTX containing the LLQTL motif (dUTX-NR) and in vitro translated ${ }^{35} \mathrm{~S}$-labelled EcR/Usp. A direct association between dUTX-NR and EcR/Usp was observed both in the presence and absence of ecdysone (Fig. 2a). This interaction was confirmed in cells where endogenous EcR immunoprecipitated with HA-dUTX both in the presence and absence of ecdysone (Fig. 2b). In addition, colocalization of EcR and dUTX was observed in vivo on salivary gland polytene chromosomes (Fig. 2c).

dUTX is required for larval salivary gland cell death. During metamorphosis the removal of obsolete larval salivary glands occurs in response to a developmental pulse of ecdysone 10-12 h relative to puparium formation (RPF). To investigate the role of $d U T X$ in ecdysone-mediated PCD in vivo, we examined the removal of the salivary glands in the loss-of-function $d U T X$ mutant, $d U T X^{1}$. dUTX is essential for development, with $d U T X$ deficiency resulting in pupal lethality, with a small percentage of 
a
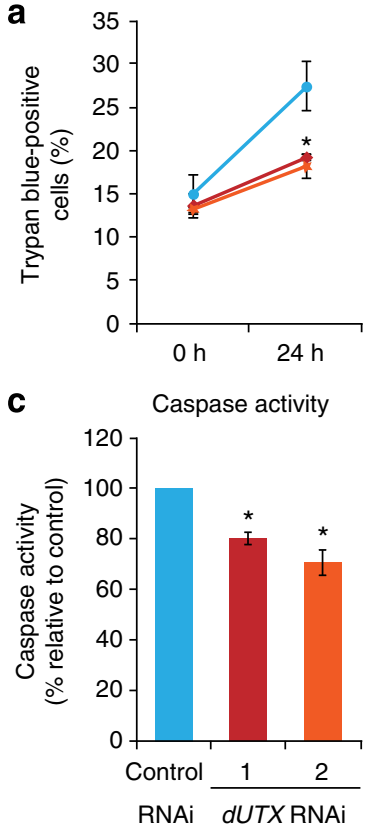

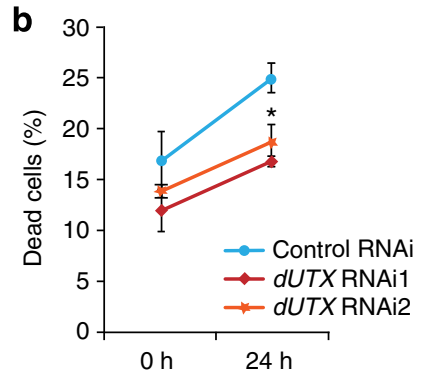

d

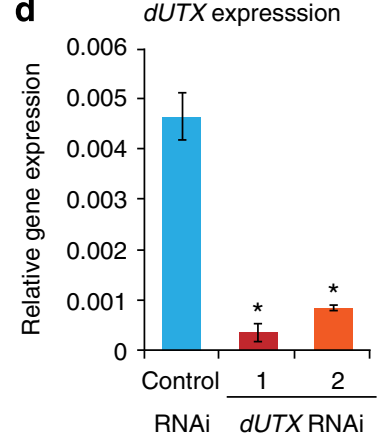

Figure 1 | dUTX regulates ecdysone-induced cell death. (a-d) RNAimediated knockdown of dUTX inhibits ecdysone-induced apoptotic cell death. SL2 cells were treated with two independent dsRNAs to dUTX or a control gene (GFP) for $48 \mathrm{~h}$ and cell death was induced with $10 \mu \mathrm{M}$ ecdysone for $24 \mathrm{~h}$. (a) The cell viability was assayed by trypan blue staining. Data are mean from three independent experiments, with error bars representing s.e.m. ${ }^{\star} P<0.05$ (Student's $t$-test). (b) Cell count and viability assay using Muse (Millipore, USA). Data are mean from three independent experiments, with error bars representing s.d. ${ }^{\star} P<0.05$ (Student's $t$-test). (c) Caspase activity was measured from total cell lysate on DEVD-AMC, represented as fold increase relative to control RNAi. Data are mean from three independent experiments, with error bars representing s.e.m. ${ }^{\star} P<0.05$ (Student's $t$-test). (d) The level of knockdown of $d U T X$ was quantificated by qPCR and normalized against ribosomal protein rp49. Relative dUTX expression levels following knockdown using the two independent dsRNA are shown $(n=3)$ with error bars representing s.d. ${ }^{\star} P<0.05$ (Student's $t$-test).

escapers that die immediately following eclosion ${ }^{23}$. In control animals the salivary glands were intact at $12 \mathrm{~h} \mathrm{RPF}$ and were degraded with only small tissue fragments remaining by $24 \mathrm{~h}$ RPF (Fig. 3a). In contrast, the degradation of the $d U T X^{1}$ salivary glands was significantly delayed, as intact salivary glands were present in many animals at $24 \mathrm{~h}$ RPF (Fig. 3a). A similar phenotype was also observed in a heteroallelic combination of $d U T X$ mutants (Supplementary Fig. S1). These results indicate that $d U T X$ is required for the ecdysone-mediated PCD of larval salivary glands during metamorphosis.

To investigate the in vivo interaction between $d U T X$ and $E c R$ we examined the RNAi-mediated knockdown of $E c R$ in the salivary glands of $d U T X^{1}$ animals. As EcR has an essential function in salivary gland removal knockdown of $E c R$ in the salivary glands results in the persistence of salivary glands well beyond the normal timing of their PCD in the controls (Supplementary Fig. S2). This strong salivary gland phenotype associated with EcR knockdown was not suitable for dosedependent genetic modification. However, we were able to examine the timing of the lethality of $d U T X^{1}$ animals where EcR levels were reduced by RNAi. Homozygous $d U T X^{1}$ animals survive past the morphological process of head eversion $(12 \mathrm{~h}$ $\mathrm{RPF}$ ) and die before eclosion. When EcR was knocked down in a $d U T X^{1}$ mutant background, the proportion of animals that

a

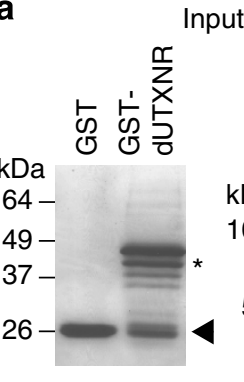

nuts

Pull down

b

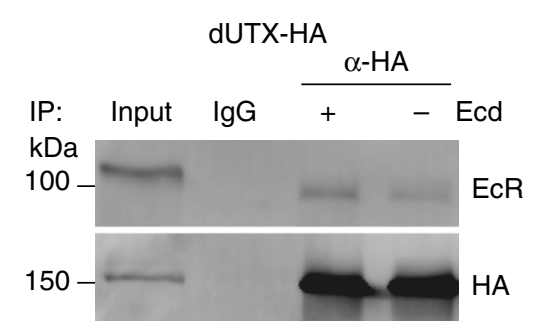

c

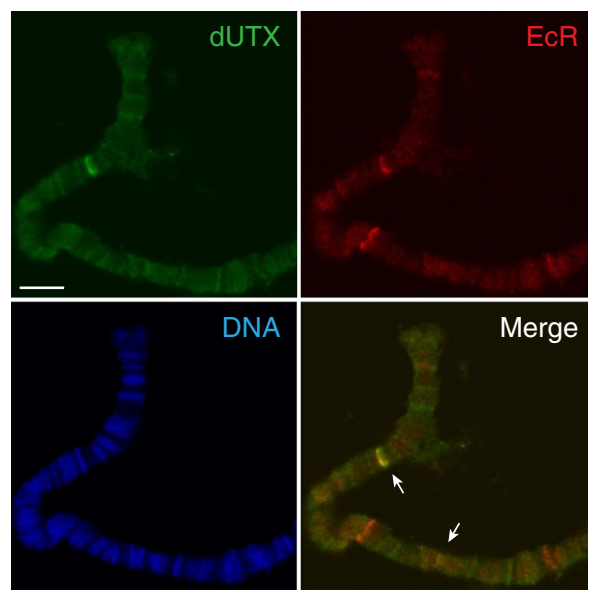

Figure 2 | dUTX directly interacts with ecdysone receptor. (a,b) dUTX directly interacts with ecdysone receptor (EcR/Usp). (a) GST and GSTdUTX-NR containing the nuclear hormone domain were used in pull-down experiments with ${ }^{35} \mathrm{~S}$-labelled EcR and Usp in the presence of ethanol control ( - ) or $10 \mu \mathrm{M}$ ecdysone ( + ). The GST input proteins are shown in Coomassie Brilliant blue-stained gel, GST is labelled with arrow head and dUTX-NR with an asterisk. The EcR and Usp inputs are shown labelled with arrows. (b) Immunoprecipitation (IP) of EcR and dUTX in SL2 cells. Cells were transfected with HA-dUTX and the cell lysates were immunoprecipitated using anti-HA or a control lgG isotype antibody. The immunoprecipitates were analysed by western blotting using the anti-EcR and anti-HA antibodies. (c) Colocalization of dUTX and EcR on salivary gland polytene chromosomes. Immunostaining of salivary glands polytene chromosome prepared from third instar larvae expressing FLAG-dUTX with dUTX (green) and EcR (red), with Hoechst-stained DNA (blue). Arrows indicate examples of colocalized bands. No signal was detected when immunostaining with secondary antibody only. Scale bar represent $5 \mu \mathrm{m}$.

survived to head eversion was significantly reduced compared with $d U T X^{1}$ alone (Table 1). This result, showing that depletion of EcR in the salivary glands of $d U T X^{1}$ enhances the lethality, implies an in vivo genetic interaction between dUTX and EcR.

dUTX regulates apoptosis and autophagy in salivary glands. A critical step required for salivary gland PCD is the induction of apoptosis, resulting in the activation of initiator caspase Dronc 
a

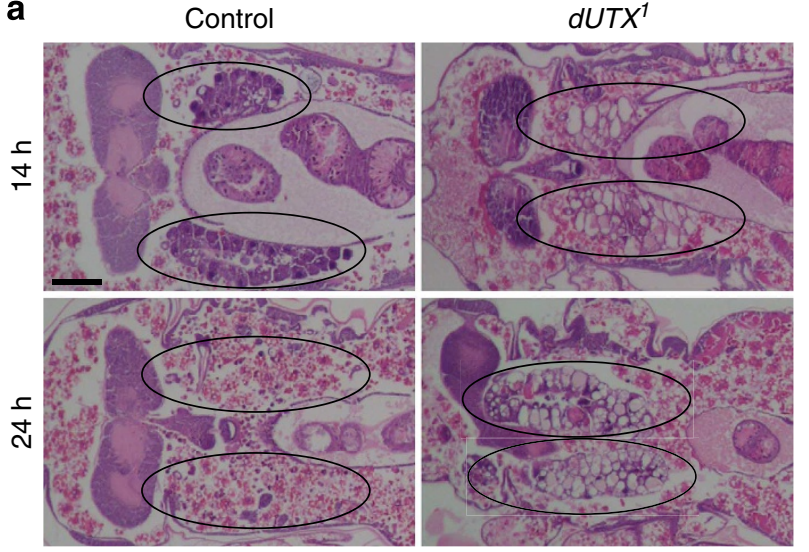

b

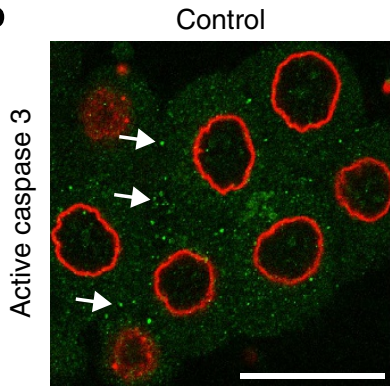

d

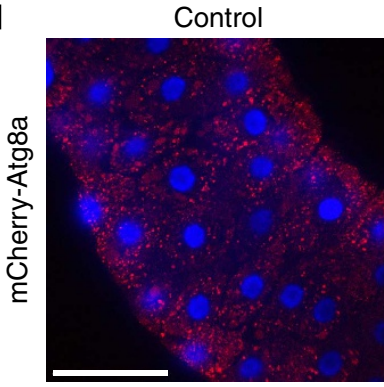

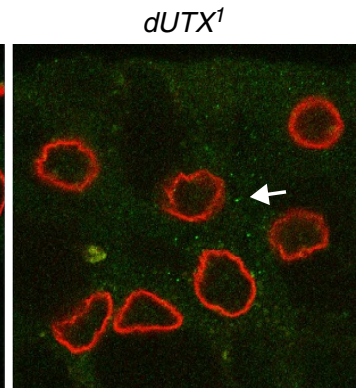

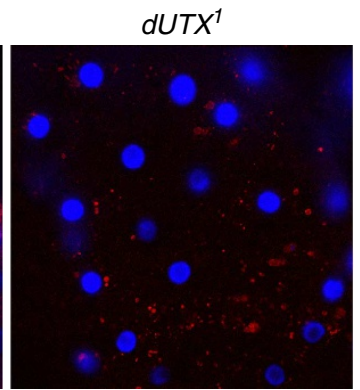

Quantitation of salivary gland degradation
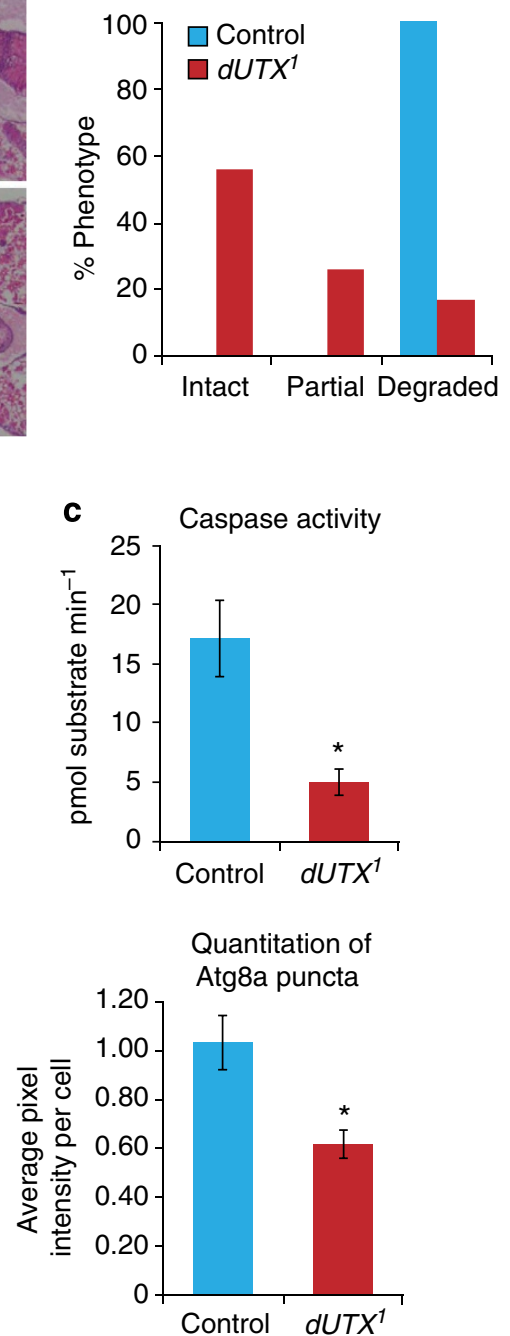

Figure 3 | dUTX is required for salivary gland cell death. (a) Histological analysis of paraffin sections at $14 \mathrm{~h}$ and $24 \mathrm{~h}$ RPF shows intact salivary glands present in $d U T X^{1}$ compared with control at $24 \mathrm{~h} \mathrm{RPF.} \mathrm{Ovals} \mathrm{indicate} \mathrm{the} \mathrm{position} \mathrm{of} \mathrm{salivary} \mathrm{glands} \mathrm{and} \mathrm{fragments.} \mathrm{Scale} \mathrm{bar} \mathrm{represent} 50 \mu \mathrm{m}$. Quantification of the salivary gland phenotypic data was at $24 \mathrm{~h}$ RPF. (b,c) Caspase staining and activity are reduced in dUTX ${ }^{7}$ salivary glands at $14 \mathrm{~h}$ RPF. (b) Cleaved caspase-3 antibody (green) and Lamin antibody (red) staining are shown in control and dUTX salivary glands at $14 \mathrm{~h}$ RPF. Scale bar represents $50 \mu \mathrm{m}$. (c) Caspase activity was measured from $14 \mathrm{~h}$ RPF control and $d U T X^{1}$ salivary glands using DEVD-AMC as a substrate. Data are mean from three independent experiments each using at least 20 salivary glands from control and dUTX for preparing cell extracts. The error bars represent s.e.m. ${ }^{\star} P$-value $<0.05$ (Student's $t$-test). (d) Autophagy puncta examined using mCherry-Atg8a (red) and nuclei stained with Hoechst (blue). Scale bar represents $100 \mu \mathrm{m}$. The quantitation of the mCherry-Atg8a puncta as the mean fluorescent pixel intensity per cell (control $\left.n=242, d U T X^{1} n=224\right)$, with error bars representing s.e.m. ${ }^{\star} P$-value $<0.05$ (Student's $t$-test).

and effector caspase Drice ${ }^{27,28}$. We examined whether the delayed degradation of the salivary glands observed in $d U T X^{1}$ was due to reduced caspase activity. Salivary glands were stained with an antibody to cleaved caspase-3 (that represents active caspases) at $14 \mathrm{~h} \mathrm{RPF}$ and compared with controls, the $d U T X^{1}$ salivary glands showed reduced immunostaining (Fig. 3b; Supplementary Fig. S3). Consistent with this, bulk caspase activity as determined by substrate cleavage, at $14 \mathrm{~h}$ RPF was significantly lower in $d U T X^{1}$ salivary glands compared with the control (Fig. 3c).

The complete removal of the larval salivary glands requires both apoptosis as well as autophagy, and blocking either pathway alone results in delayed degradation ${ }^{26}$. To determine whether the delayed removal of salivary glands in $d U T X^{1}$ was also due to reduced autophagy we examined the levels of mCherry-Atg8a puncta formation ${ }^{40}$. In the control salivary glands, high levels of puncta were clearly evident at $14 \mathrm{~h}$ RPF; however, $d U T X^{1}$ salivary glands showed reduced puncta at the same time (Fig. 3d). This suggests that in addition to impaired caspase activity there is also a reduction in autophagy, coinciding with delayed removal of the salivary glands in $d U T X$ mutant animals.

As removal of the salivary glands is triggered by ecdysone, the defects observed in the $d U T X$ mutant animals could potentially be due to a more general defect in ecdysone synthesis and levels. If dUTX has a role in the production of ecdysone, it is likely to be at the level of transcription of ecdysone biosynthesis genes. We examined the expression of genes involved in ecdysone biosynthesis and found that their transcript levels in $d U T X^{1}$ animals were comparable to the transcript levels in the control 
animals at the same stage (Supplementary Fig. S4a). Additionally, the levels of ecdysone assessed at $12 \mathrm{~h} \mathrm{RPF}$ were unaffected in the $d U T X^{1}$ animals (Supplementary Fig. S4b), suggesting that dUTX does not affect ecdysone production or levels. These findings are

\section{Table 1 | Combined loss of $d U T X$ and EcR increases lethality of $d U T X$}

\begin{tabular}{|c|c|c|c|c|c|c|}
\hline Genotype $^{\star}$ & dUTX ${ }^{1}$ & yOKrGFP & CyO & Total & $\chi_{2 \mathrm{df}}^{2}$ & ue \\
\hline & $30(34)$ & & & & 年 & \\
\hline U & $46(60)$ & $53(60)$ & $81(60)$ & 180 & 11.433 & \\
\hline
\end{tabular}

\begin{tabular}{llllll}
\hline & dUTX $^{\mathbf{1}}$ & & \multicolumn{2}{c}{$\boldsymbol{\chi}^{\mathbf{2}_{\text {1df }}}$} \\
\hline+ & $102(99)$ & $96(99)$ & 198 & 0.182 & 0.669 \\
\hline
\end{tabular}

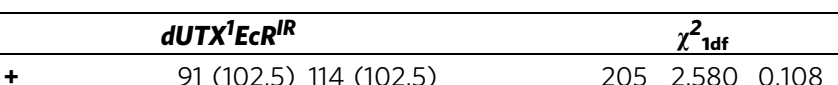

$\chi^{2}$ analysis of the number of progeny obtained from $d U T X^{1} /$ CyOActGFP; Sgs3-GAL4 crossed to $d U T X^{1} /$ CyOKrGFP or crossed to dUTX UAS-EcR ${ }^{R} /$ CyOKrGFP with 2 degrees of freedom at $12 \mathrm{~h}$ RFP. The control crosses were Sgs3-Gal4-crossed to either $d U T X^{\top} / C^{\prime} y O K r G F P$ or crossed to $d U T X^{7}$ $U A S-E C R^{R} /$ CyOKrGFP with 1 degree of freedom.

$\mathrm{df}$ is the number of the degrees of freedom. $P$ is the statistical significance value obtained from the $\chi^{2}$ test.

${ }^{*}$ All genotypes also contain salivary gland driver Sgs3-GAL4/+

†The observed numbers from the crosses are shown with the expected numbers for each

genotype, based on Mendelian ratios of 1:1:1 or 1:1 for control crosses, shown in parentheses. consistent with the observations that $d U T X^{1}$ animals undergo morphological changes during metamorphosis, including pupariation ( $0 \mathrm{~h} \mathrm{RPF})$ and head eversion (12 h RPF), indicating that other ecdysone-regulated processes are not affected. As stated above, the majority of $d U T X^{1}$ animals fail to progress through later developmental stages and $\mathrm{die}^{23}$.

dUTX regulates expression of apoptosis and autophagy genes. As an H3K27me3 demethylase, dUTX may be recruited by EcR/ Usp to the promoters of specific genes to remove the repressive epigenetic mark. Given the reduction in caspase activity observed in $d U T X$ mutant salivary glands, we investigated whether the expression of apoptosis genes was altered in the absence of $d U T X$. Previous studies have shown that ecdysone regulates transcription of dronc, drice, dark and $r p r$ to induce salivary gland $\mathrm{PCD}^{31-36}$ An examination of the transcriptional profile of dronc, drice, dark and $r p r$ showed similar transcript levels for these genes in control and $d U T X^{1}$ salivary glands prior to cell death at $6 \mathrm{~h}$ RPF (Fig. 4a). Following initiation of cell death at $14 \mathrm{~h}$ RPF, dronc, drice and rpr expression was induced in the control salivary glands; however in the $d U T X^{1}$ salivary glands there were significantly lower transcript levels of these genes (Fig. 4a).

As there was also a reduction in autophagy levels in the $d U T X^{1}$ salivary glands, we investigated whether the expression of autophagy genes were altered in the absence of dUTX. The
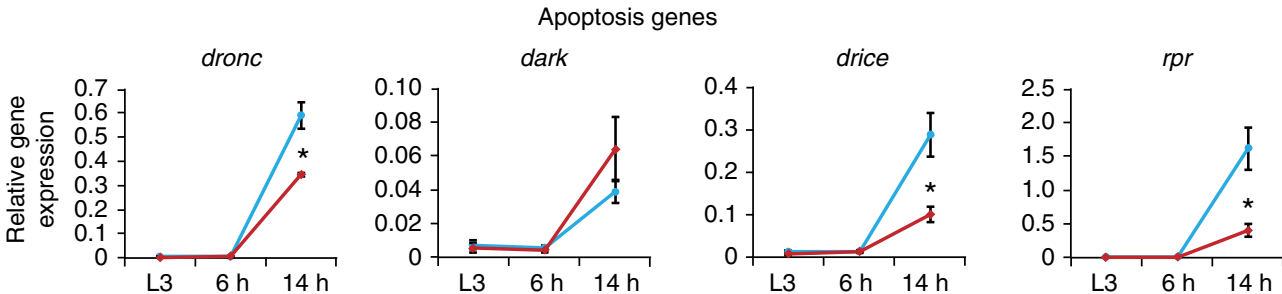

b
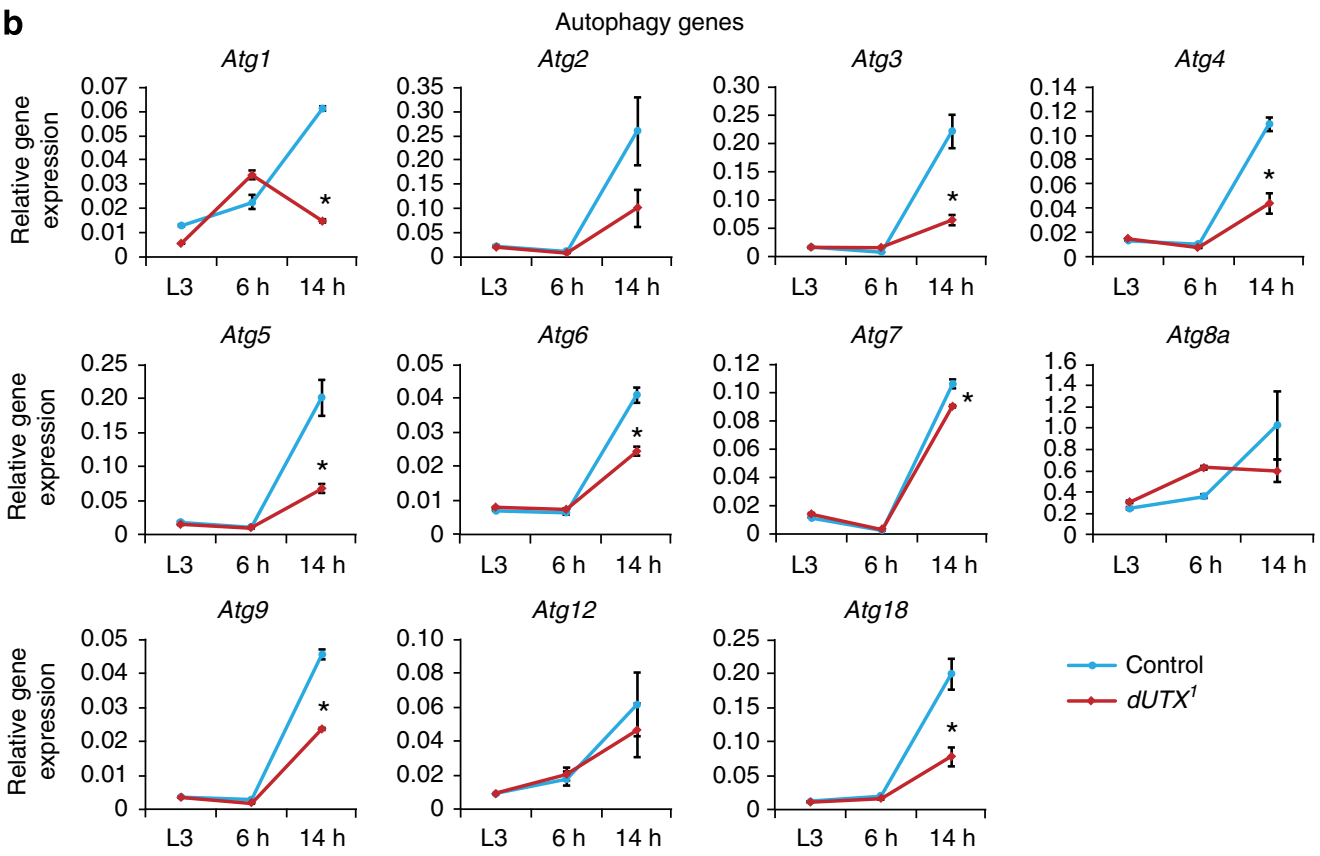

Figure 4 I dUTX regulates ecdysone-mediated transcription of PCD and Atg genes. (a) Levels of dark, dronc, drice and rpr transcripts were measured from third instar larvae (L3), $6 \mathrm{~h}$ and $14 \mathrm{~h}$ RPF salivary glands from control (blue) and $d U T X^{1}$ (red) by qPCR and expressed relative to the internal control gene rp49. (b) The transcript levels of Atg genes relative to the internal control gene rp49 in the salivary glands of control (blue) and dUTX (red) were analysed by qPCR at L3, $6 \mathrm{~h}$ and $14 \mathrm{~h}$ RPF. Data in all panels are expressed as means from three independent experiments, with error bars representing s.e.m. ${ }^{\star} P<0.05$ (Student's $t$-test). 
transcript levels of several Autophagy-related (Atg) genes have been shown to increase in salivary glands at $14 \mathrm{~h} \mathrm{RPF}^{29,30}$. Prior to the induction of PCD at $6 \mathrm{~h} \mathrm{RPF}$ the transcript levels of the Atg genes examined remained at low levels in $d U T X^{1}$ similar to the control (Fig. 4b). However, at $14 \mathrm{~h} \mathrm{RPF}$ there was significantly reduced expression of several Atg genes, including Atg1, Atg3, Atg4, Atg5, Atg6, Atg7, Atg9 and Atg18, in UUTX $^{1}$ compared with control where the expression of Atg genes was higher (Fig. 4b). The lower levels of apoptosis and autophagy gene transcripts in $d U T X^{1}$ salivary gland at the time of PCD suggests that dUTX plays an important role in maintaining the correct temporal regulation of the transcriptional response to ecdysone.

dUTX is recruited to apoptosis and autophagy gene promoters. The findings above imply that dUTX is recruited to cell death gene promoters to control the timing of the hormone-mediated removal of the salivary glands. To examine the recruitment of dUTX to the promoters of the ecdysone-responsive genes, we performed chromatin immunoprecipitation (ChIP) assays using SL2 cells transfected with tagged dUTX following treatment with ecdysone. dUTX was recruited to the promoter of apoptosis genes dark, dronc, drice and $r p r$ (Fig. 5a). Furthermore, we observed recruitment of dUTX on several Atg gene promoters, including Atg1, Atg2, Atg3, Atg4, Atg5, Atg6, Atg7, Atg8a, Atg9 and Atg18, but not on the Atg12 promoter (Fig. 5b). This is consistent with the expression profile of these genes during salivary gland cell death, where $d U T X^{1}$ did not show significantly different levels of Atg12 transcripts. The demethylase activity of dUTX targets H3K27me3 so we would predict that in the absence of $d U T X$ the promoters of the apoptosis and autophagy genes would maintain higher levels of H3K27me3. In dUTX knockdown cells ChIP analysis using H3K27me3 shows enrichment of H3K27me3 on the promoters of several apoptosis and autophagy genes (Fig. 5c). The promoters of apoptosis genes dark and $r p r$ as well as autophagy genes, Atg1, Atg2, Atg7, Atg9 and Atg18 showed significantly increased $\mathrm{H} 3 \mathrm{~K} 27 \mathrm{me}$. The minor discrepancies detected between $d U T X$ knockdown cells and $d U T X^{1}$ animals
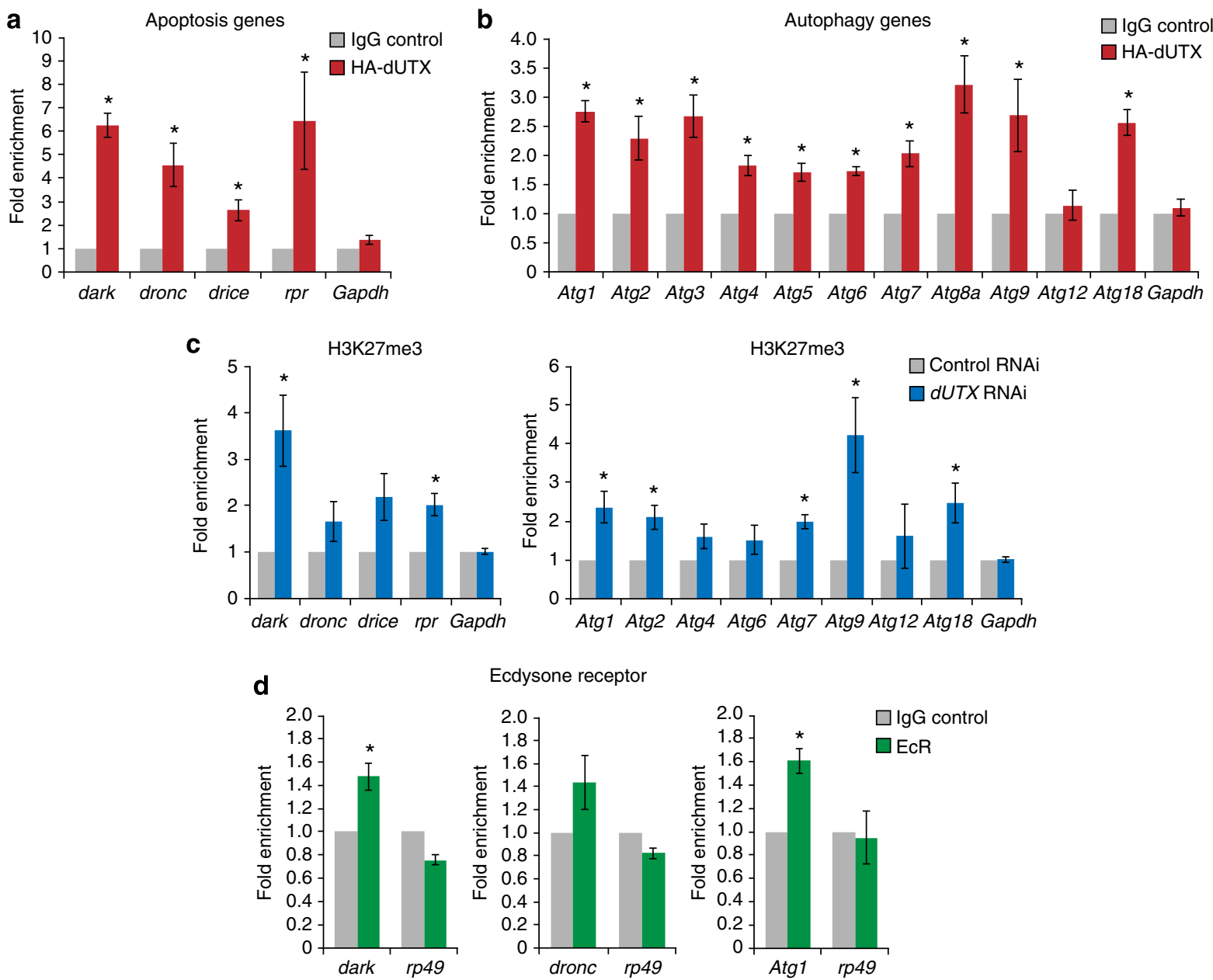

Ecdysone receptor
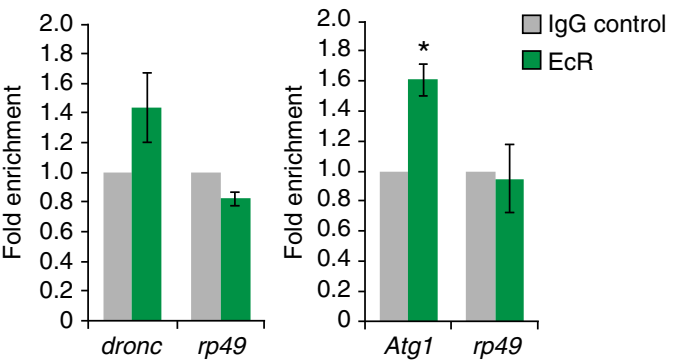

Figure 5 | dUTX is recruited to the promoters of apoptosis and Atg genes. (a,b) The occupancy of dUTX on the promoter regions of apoptosis and Atg genes was detected by chromatin immunoprecipitation (ChIP). SL2 cells overexpressing HA-dUTX were chromatin-immunoprecipitated with anti-HA and anti-GFP (IgG control) antibodies following ecdysone treatment. qPCR was used to assess the binding to the promoters of dark, dronc, drice and rpr (a) and Atg1, Atg2, Atg3, Atg4, Atg5, Atg6, Atg7, Atg8a, Atg9, Atg12 and Atg18 (b), expressed relative to the internal control gene Gapdh. Results are shown as the fold enrichment of \% input compared with IgG control. (c) The abundance of H3K27me3 on the promoters of apoptosis and autophagy genes detected by ChIP. SL2 cells knocked down for dUTX were chromatin-immunoprecipitated with anti-H3K27me3 and anti-GFP (IgG control) antibodies following ecdysone treatment. Results are shown as the fold enrichment of \% input compared with RNAi control. (d) The occupancy of EcR on the promoter regions of dark, dronc and Atg1 by ChIP expressed relative to the internal control gene rp49. Chromatin was immunoprecipitated with anti-EcR-B1 and anti-GFP (IgG control) antibodies from SL2 cell following ecdysone treatment. Results are shown as the fold enrichment of \% input compared with lgG control. Data in all panels are expressed as means from three independent experiments, with error bars representing s.e.m. ${ }^{\star} P<0.05$ (Student's $t$-test). 
most likely reflect the molecular differences between ecdysoneinduced cell death in the cell line compared with that in vivo.

While the expression data show that the transcript levels of Atg genes increase following ecdysone, it is not known if this is due to the direct binding of EcR/Usp to the promoters or secondary transcription factors. As Atg1 is a key upstream regulator of autophagy, we examined the Atg1 promoter sequence for potential EcR consensus DNA-binding sequence and identified a region +335 nucleotides from the transcription start site. Given our findings that dUTX is recruited to the Atg1 gene promoter we predicted that EcR would also be present at this promoter. Consistent with previous studies, following ecdysone treatment, there was an increase in EcR-B1 recruitment on the dronc and dark promoters (Fig. 5d). In addition, using primers spanning the potential EcR-binding sequence we also detected the recruitment of EcR-B1 to the Atg1 promoter (Fig. 5d). Taken together, these data provide a model whereby EcR/Usp recruits dUTX to the promoters of apoptosis and autophagy genes, required for the temporal induction of transcription in response to ecdysone during salivary gland PCD. This is an important finding, as UTX family members have not previously been implicated in hormone-mediated transcription of apoptosis and autophagy genes.

dUTX regulates expression of E93. In addition to the direct regulation of apoptosis gene expression by EcR/Usp, a transcriptional hierarchy is triggered in response to ecdysone whereby EcR/Usp (along with the $\beta F T Z-F 1$ orphan nuclear receptor) directly activates transcription of the early genes Broad Complex $(B R-C), E 74$ and $E 93^{41-43}$. These early genes encode transcription factors and activate transcription of late genes to establish tissuespecific outcomes ${ }^{44}$. While BR-C, E74 and E93 are required for salivary gland PCD, E93 functions as a stage-specific regulator of cell death and BR-C and E74 have wider developmental roles ${ }^{25,44-46}$. In particular, the transcription of $r p r$ and dronc requires ecdysone-regulated early genes $\mathrm{BR}-\mathrm{C}$ and E93 and not E74 (ref. 24). Given this we examined the expression of $B R-C$ and E93 in $d U T X^{1}$ salivary glands. The levels of $E 93$ were significantly decreased in $d U T X^{1}$ salivary glands at $14 \mathrm{~h}$ RPF while there was no significant change in the $B R-C$ transcript levels compared with control (Fig. 6a). Furthermore, ChIP analysis revealed that dUTX was recruited to the E93 promoter and dUTX knockdown resulted in increased $\mathrm{H} 3 \mathrm{~K} 27 \mathrm{me} 3$ (Fig. 6b). This suggests that dUTX plays a distinct role in both the early and the late transcriptional response to ecdysone.

Demethylase activity of dUTX is required for cell death. Consistent with the H3K27me3 demethylase activity of dUTX the level of $\mathrm{H} 3 \mathrm{~K} 27 \mathrm{me} 3$ was increased in $d U T X^{1}$. Mosaic clonal analysis revealed increased $\mathrm{H} 3 \mathrm{~K} 27 \mathrm{me} 3$ immunostaining in $d U T X^{1}$ clones compared with the neighbouring wild-type cells (Fig. 7a), with only a small decrease in H3K4mel (Supplementary Fig. S5). The total levels of H3K27me3 were similarly increased in whole $d U T X$ mutant salivary glands compared with the control (Fig. 7b). To determine whether the demethylase function of dUTX was required for salivary gland PCD, we expressed wildtype or JmjC domain mutant dUTX specifically in the salivary gland in a $d U T X^{1}$ background. The dUTX mutant JmjC domain $\left(\mathrm{JmjC}^{\star}\right.$ ) lacks two residues (His883 and Glu885) critical for its catalytic function ${ }^{23}$. While expression of the wild-type dUTX partially rescued the delayed salivary gland degradation of the dUTX mutant, the expression of the catalytically inactive dUTX did not (Fig. 7c). These findings support the role of dUTX catalytic activity in regulation of salivary gland PCD. a
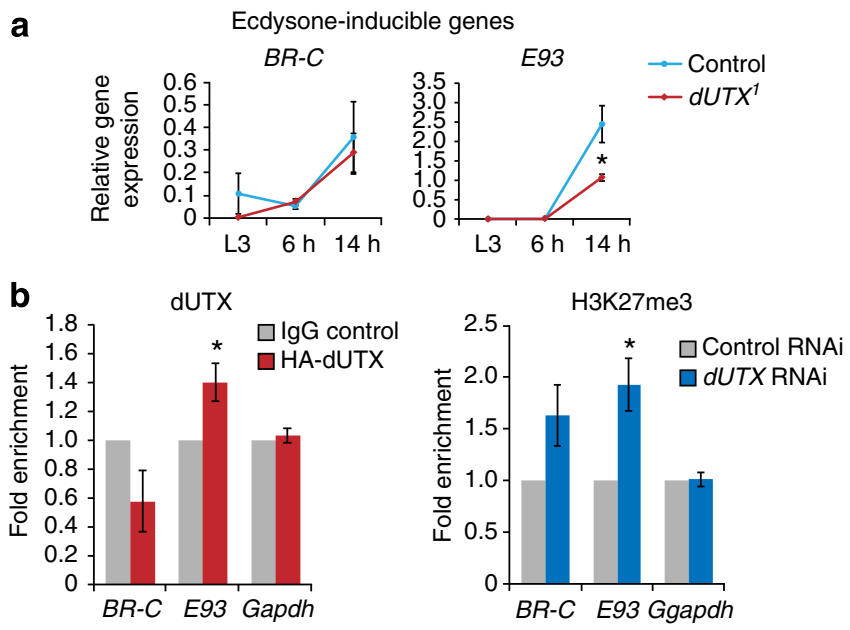

Figure 6 | dUTX regulates ecdysone-induced gene E93. (a) Levels of $B R-C$ and $E 93$ transcripts were measured in salivary glands isolated from third instar larvae (L3), $6 \mathrm{~h}$ and $14 \mathrm{~h}$ RPF control (blue line) and $d U T X^{\top}$ (red line) using QPCR and expressed relative to the internal control gene rp49. (b) The occupancy of dUTX on the promoter regions of $B R-C$ and $E 93$ genes detected by ChIP. SL2 cells overexpressing HA-dUTX were chromatinimmunoprecipitated with anti-HA and anti-GFP (IgG control) antibodies following ecdysone treatment. Results are shown as the fold enrichment of $\%$ input compared with IgG control. In the right hand panel abundance of $\mathrm{H} 3 \mathrm{~K} 27 \mathrm{me} 3$ on the promoters of $B R-C$ and $E 93$ genes as detected by ChIP is shown. SL2 cells knockdown for dUTX (dUTX RNAi) were chromatinimmunoprecipitated with anti-H3K27me3 and rabbit IgG (control) antibodies following ecdysone treatment. Results are shown as the fold enrichment of \% input compared with RNAi control. Data in all panels are expressed as means from three independent experiments, with error bars representing s.e.m. ${ }^{\star} P<0.05$ (Students $t$-test).

\section{Discussion}

UTX function is known to be critical in mammalian embryonic development and somatic and germ cell reprogramming. Here we found a novel role for $d U T X$ in steroid hormone-mediated cell death during development. Our study provides the novel observation that dUTX, together with nuclear hormone receptor $\mathrm{EcR} / \mathrm{Usp}$, is capable of regulating gene expression both spatially and temporally in a hormone-dependent manner. UTX gene mutations are frequently observed in malignancies including lethal castration-resistant prostate cancer ${ }^{18,47}$, although a role for UTX in androgen receptor-mediated transcription has not yet been identified 8 . Our study indicates that UTX is a good candidate to extend the investigation to examine the role of UTX in coordinating nuclear hormone receptor-regulated gene expression, particularly in androgen receptor-mediated transcription during mammalian development and hormonedependent cancers.

The complete degradation of larval salivary glands during metamorphosis utilizes both apoptosis and autophagy and by coordinately controlling the expression of critical genes in these two distinct biological pathways, dUTX ensures timely removal of salivary glands in response to temporal ecdysone pulse. The majority of studies addressing induction of autophagy have focused upon autophagosome formation and protein degradation. The transcriptional regulation of autophagy induction remains poorly understood. Indeed, several Atg genes are transcriptionally upregulated following autophagy induction; however, the molecular pathways are only beginning to be revealed. For example, the master gene controlling lysosomal biogenesis, transcription factor EB, coordinates the expression of both autophagy and 
a

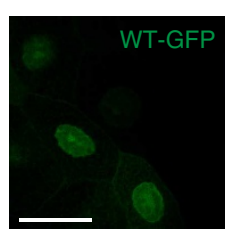

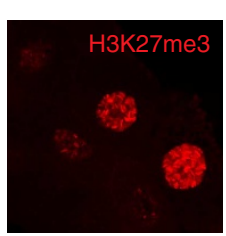

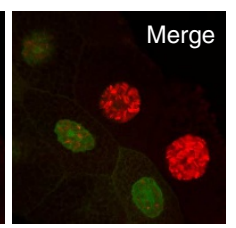

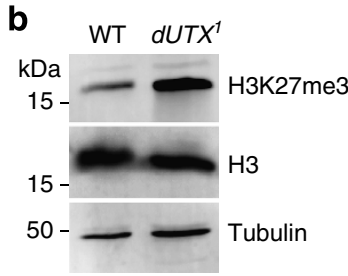

C

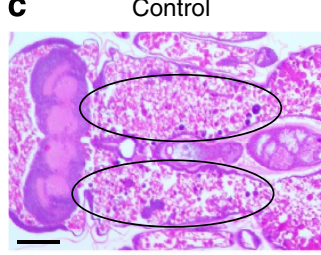
dUTX'; $d U T X^{W T}$

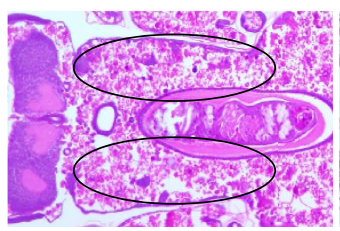

$d U T X^{1}$

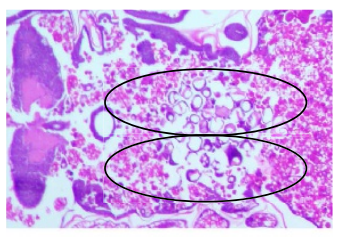

$d U T X^{1} ; d U T X^{J m j *}$

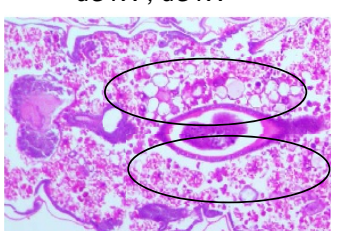

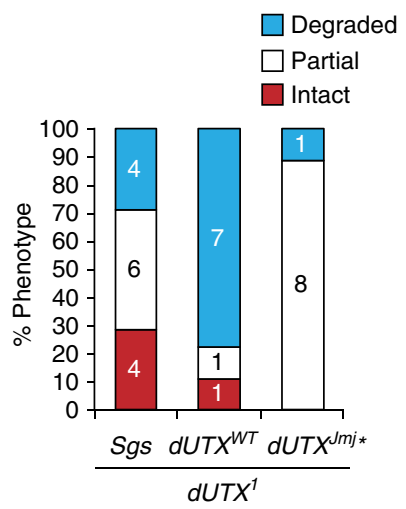

d

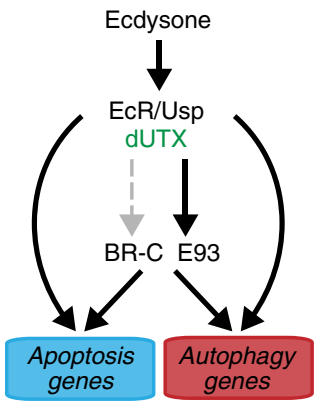

Figure 7 | dUTX demethylase activity is required for cell death. (a) Mosaic clones of $d U T X^{7}$ (hsFLP; ubi-GFP FRT4OA/dUTX ${ }^{1}$ FRT4OA) from third instar larval salivary glands with wild-type cells marked by nuclear-localized GFP (green). dUTX clones have high levels of H3K27me3 (red) compared with the neighbouring control cells marked by GFP. Scale bar represents $50 \mu \mathrm{m}$. (b) Western blot analysis of the level of H3K27me3 in control and dUTX larval salivary glands. Alpha-tubulin and $\mathrm{H} 3$ serve as loading controls. (c) Expression of dUTX wild-type (dUTXWT) and dUTX catalytic mutant (dUTX ${ }^{\text {Imj }}$ ) in the salivary glands (using Sgs3-GAL4 driver) of $d U T X^{1}$. Histological analysis of paraffin sections at $24 \mathrm{~h}$ RPF shows intact salivary glands present in $d U T X^{1}$ and in $d U T X^{\top} ; d U T X^{J m j^{\star}}$ compared with control and $d U T X^{\top} ; d U T X^{W T}$ at $24 \mathrm{~h}$ RPF. Ovals indicate the position of salivary glands and fragments. Scale bar represent $50 \mu \mathrm{m}$. Quantification of the salivary gland phenotypic data at $24 \mathrm{~h}$ RPF is shown on the right. (d) A schematic representation of the role of dUTX in ecdysone-mediated transcriptional regulation of cell death genes, directly or via E93.

lysosomal genes to induce autophagy in response to starvation ${ }^{48}$. More recently, induction of autophagy has been linked to reduced histone $\mathrm{H} 4$ lysine 16 acetylation (H4K16ac) through downregulation of the histone acetyltransferase hMOF ${ }^{49}$. Downregulation of $\mathrm{H} 4 \mathrm{~K} 16$ deacetylation was associated with the downregulation of several Atg genes, whereas antagonizing H4K16ac downregulation upon autophagy induction resulted in cell death. The study indicates that a specific histone modification during autophagy modulates the expression of Atg genes, and is important for survival versus death responses upon autophagy induction ${ }^{49}$. Our work now describes dUTX as another regulator of autophagy and cell death in the context of developmental PCD and in concert with the steroid hormone response. Future studies to understand the complex nuclear events regulating both repression and induction of autophagy gene expression in response to particular signals will be important.

Despite the opposing roles of $\mathrm{H} 3 \mathrm{~K} 27$ and $\mathrm{H} 3 \mathrm{~K} 4$ methylation in transcriptional regulation, UTX has been identified in association with $\mathrm{H} 3 \mathrm{~K} 4$ methyltransferase and to play demethylase-independent functions ${ }^{23,18,19}$. Our study suggests that the demethylase activity of dUTX is necessary for hormone-mediated cell death. The nuclear hormone receptor response to ecdysone initiates a hierarchical transcription cascade by induction of transcription factors, including BR-C, E74 and E93. These transcription factors drive expression of downstream genes including cell death genes. Our data show that dUTX regulates E93 and suggests that this HDM can regulate cell death both directly, through the transcription of apoptosis and autophagy genes through direct recruitment via EcR/ Usp, as well as indirectly through key transcription factor E93 (Fig. 7d). This additional level of regulation through the stagespecific transcription factor E93 may provide temporal control of ecdysone response during metamorphosis.
The role of autophagy in cell death is a matter of considerable debate as autophagy is generally a cell survival mechanism in response to cellular stress and nutrient limitations ${ }^{50}$. Studies in Drosophila have provided perhaps some of the strongest evidence for a role of autophagy in developmental cell death in vivo. Our data presented in this paper demonstrating coordinate regulation of both key apoptosis and autophagy genes by a single histonemodifying enzyme further provide genetic and molecular evidence linking autophagy and apoptosis in PCD during metamorphosis.

\section{Methods}

Cell culture and RNA interference. SL2 cells were cultured in Schneider's medium (GIBCO, Life Technologies, Carlsbad, CA, USA) supplemented with 10\% FBS and $1 \%$ penicillin-streptomycin at $28^{\circ} \mathrm{C}$. Ecdysone (Sigma-Aldrich, St Louis, MO, USA) was used at $10 \mu \mathrm{M}$. For dsRNAi template 1 the region $1-531 \mathrm{bp}$ and for dsRNAi template 2 the region 2,935-3,405 bp of dUTX-RA was cloned into pGEM-T (Promega, Madison, WI, USA) and double-stranded RNAs (dsRNA) were generated by in vitro transcription kit MEGAscript (Ambion, Life Technologies, Carlsbad, CA, USA) using T7/Sp6 polymerase. Control dsRNAi construct containing GFP in pGEM-T was as described ${ }^{51}$. For RNAi treatment, SL2 cells were washed once and resuspended in serum-free medium at $2 \times 10^{6}$ cells per $\mathrm{ml}$. dsRNA $(25 \mu \mathrm{g})$ was added to $1 \mathrm{ml}$ of cell suspension and incubated for $3 \mathrm{~h}$ at $28^{\circ} \mathrm{C}$. After the incubation, $2 \mathrm{ml}$ complete medium was added to the cells and cultured for another $48 \mathrm{~h}$ before harvesting the cells for RNA extraction or caspase assays. For cell viability SL2 cell suspension $(20 \mu \mathrm{l})$ in Schneider media was incubated with equal volume of trypan blue and counted on haemocytometer or with $180 \mu \mathrm{l}$ of Muse Count \& Viability Reagent for $5 \mathrm{~min}$ at room temperature and analysed using Muse (Millipore, Billerica, MA, USA) instrument. For expression of HA-tagged dUTX in SL2 cells, dUTX was cloned into the Drosophila Gateway vector collection pAWH (containing Actin5C promoter with C-terminal $3 \times$ HA epitopes) according to the Gateway Technology manual (Invitrogen, Life Technologies, Carlsbad, CA, USA). Briefly, dUTX was amplified using $d U T X-F 5^{\prime}$-CACCATGGGGCAACTAACTTTCGAAG- $3^{\prime}$ and $d U T X-\mathrm{R}$ $5^{\prime}$-CTGAGTGTATGCTAGTCGTTGG-3' primers and cloned into $\mathrm{pENTR/SD/}$ D-TOPO (Invitrogen). The resulting entry clone, pENTR-dUTX, was then 
recombined with pAWH destination vector using Gateway LR Clonase Enzyme Mix (Invitrogen).

Quantitative real-time PCR. Quantification of gene expression and the level of RNAi knockdown of various lines was performed by qPCR. Total RNA was isolated from Drosophila salivary glands and SL2 cells using TRIzol reagent (Invitrogen). cDNA was synthesized using High Capacity cDNA Reverse Transcription Kit (Applied Biosciences, Life Technologies) and oligo dT primer (Geneworks, Thebarton, SA, Australia), with $1 \mu \mathrm{g}$ of total RNA. Briefly, qPCR was performed using $2 \mu \mathrm{l}$ of cDNA and $5 \mu \mathrm{l}$ of $\mathrm{RT}^{2}$ Real-Time SYBR Green/ROX PCR MasterMix (Qiagen, Valencia, CA, USA) in $10 \mu \mathrm{l}$ reactions on a Rotor-Gene 6000 (Corbett Research, Mortlake, NSW, Australia). Reactions were performed in triplicate and the mRNA expression levels were normalized against the internal control gene rp49 using the $\Delta \Delta \mathrm{CT}$ method. Primer sets used are described in Supplementary Table S1.

Caspase activity assays. The caspase activity assays were performed as previously described $^{52}$, using $70 \mu \mathrm{g}$ of salivary gland protein lysate or $50 \mu \mathrm{g}$ of total protein lysate from SL2 cells with $100 \mu \mathrm{M}$ of DEVD-AMC (MP Biomedicals, Solon, OH, USA) made up to a final volume of $100 \mu \mathrm{l}$ in caspase assay buffer $(50 \mathrm{mM}$ HEPES, $\mathrm{pH} 7.5,100 \mathrm{mM} \mathrm{NaCl}, 1 \mathrm{mM}$ EDTA, $0.1 \%$ CHAPS, $10 \%$ sucrose, $5 \mathrm{mM}$ DTT, $0.5 \%$ TritonX-100, 4\% glycerol with protease inhibitor mix (Roche, Indianapolis, IN, USA)). The fluorescence was measured at $30 \mathrm{~min}$ intervals over a $3 \mathrm{~h}$ time course using a FluoStar spectrophotometer (BD Biosciences, Franklin Lakes, NJ, USA) (excitation, $385 \mathrm{~nm}$; emission, $460 \mathrm{~nm}$ ). The change in DEVD-AMC fluorescence over time was plotted to determine the linear slope of the line that was multiplied by the conversion factor and the reaction volume expressed as pmol substrate per min (ref. 52). The conversion factor was calculated as the inverse of the slope of the standard curve obtained by plotting the fluorescence reading of the serial dilutions $(5,10,25,50$ and $100 \mu \mathrm{M})$ of DEVD-AMC ${ }^{52}$

GST pull-down. For the expression of recombinant dUTX-NR region as GST fusion protein, the sequence corresponding to amino acids $820-1,136$ was cloned into pGEX-2T using primers for dUTX-NR $5^{\prime}$-AGGCAAGGATCCAGGTTCGGG ACCAACGTGGACCTCTCC- ${ }^{\prime}$ and $5^{\prime}$-AGGCAAGGATCCCTGAGTGTAT GCTAGTCCTTGGG-3'. To generate ${ }^{35}$ S-labelled EcR and Usp, the pCMX-EcR ${ }^{53}$ and pCMX-Usp ${ }^{53}$ templates were used with the TNT Coupled Reticulocyte Lysate System (Promega) in the presence of ${ }^{35}$ S-Methionine (MP Biomedicals).

Chromatin immunoprecipitation. For chromatin immunoprecipitation (ChIP), $\sim 6 \times 10^{6}$ SL2 cells were fixed in $1 \%$ formaldehyde at room temperature for $10 \mathrm{~min}$, then glycine was added to a final concentration of $0.125 \mathrm{M}$ and incubated for a further $5 \mathrm{~min}$. Cells were washed thrice in ice-cold PBS and lysed in cell lysis buffer (1\% SDS, $10 \mathrm{mM}$ EDTA, $50 \mathrm{mM}$ Tris- $\mathrm{HCl}$ ( $\mathrm{pH} 8.1$ ) and complete protease inhibitors cocktails (Roche)). The cell lysate was sonicated for $25 \mathrm{~min}$ (30 s on/off) using Bioruptor (Diagenode, Liège, Belgium) to shear the chromatin into 200-500 bp fragments. The chromatin solution was then clarified and pre-cleared by incubating with protein $\mathrm{G}$ agarose beads for $1 \mathrm{~h}$ at $4^{\circ} \mathrm{C}$. Two micrograms of antibodies anti-HA, anti-EcR-B1 (AD4.4, Developmental Studies Hybridoma Bank, Iowa, IA, USA), anti-H3K27me3 (Abcam, Cambridge, UK) and anti-GFP (Roche) were used for each ChIP reaction and incubated overnight at $4{ }^{\circ} \mathrm{C}$. The protein $\mathrm{G}$ beads were prepared by incubating with BSA and salmon sperm DNA before adding into the ChIP reactions and incubated for $2 \mathrm{~h}$ at $4{ }^{\circ} \mathrm{C}$. The beads were washed once in low-salt wash buffer $(0.1 \%$ SDS, $1 \%$ Triton X-100, 2 mM EDTA, $20 \mathrm{mM}$ Tris- $\mathrm{HCl}(\mathrm{pH} 8.1), 150 \mathrm{mM} \mathrm{NaCl})$, high-salt wash buffer $(0.1 \%$ SDS, $1 \%$ Triton X-100, 2 mM EDTA, 20 mM Tris- $\mathrm{HCl}$ (pH 8.1), $500 \mathrm{mM} \mathrm{NaCl}), \mathrm{LiCl}$ wash buffer $(250 \mathrm{mM} \mathrm{LiCl}, 1 \% \mathrm{NP} 40,1 \%$ deoxycholate, $1 \mathrm{mM}$ EDTA, $10 \mathrm{mM}$ Tris-HCl $(\mathrm{pH} 8.1))$ and twice in TE buffer ( $10 \mathrm{mM}$ Tris-HCl, $1 \mathrm{mM}$ EDTA (pH 8.0)). The immunoprecipitated DNA was eluted from the beads in freshly prepared elution buffer $\left(0.1 \mathrm{M} \mathrm{NaHCO}_{3}\right.$ and $1 \%$ SDS). The eluates were then reverse-crosslinked by incubating at $65^{\circ} \mathrm{C}$ overnight. The DNA was purified by phenol-chloroform extraction and ethanol precipitation. The precipitated DNA was then analysed by qPCR and the promoter recruitment is expressed as the fold enrichment over control IgG normalized by the input DNA. Primer sequences for qPCR are listed in Supplementary Table S2.

Co-immunoprecipitation. Cells transfected with pAWH-dUTX-HA were lysed in cell lysis buffer (20 mM Tris- $\mathrm{HCl}$ (pH 7.8), $100 \mathrm{mM} \mathrm{NaCl}, 0.5 \%$ NP40, 5\% glycerol, $1 \mathrm{mM}$ EDTA and complete protease inhibitors cocktails from Roche) for $15 \mathrm{~min}$ on ice. The nuclear fraction was pelleted by centrifugation at 13,000 r.p.m. for $30 \mathrm{~s}$ at $4{ }^{\circ} \mathrm{C}$ and lysed in nuclear lysis buffer $(20 \mathrm{mM}$ Tris- $\mathrm{HCl}$ (pH 7.4), $400 \mathrm{mM} \mathrm{NaCl}$, $7.5 \mathrm{mM} \mathrm{MgCl}_{2}, 0.2 \mathrm{mM}$ EDTA, $0.1 \mathrm{mM}$ EGTA, $1 \mathrm{mM}$ DTT with complete protease inhibitors cocktail (Roche)) for $45 \mathrm{~min}$ at $4{ }^{\circ} \mathrm{C}$. Both cytoplasmic and nuclear lysates were combined and $1 \mathrm{mg}$ of total proteins was immunoprecipitated with $10 \mu \mathrm{g}$ anti-HA antibodies (Roche, cat. no. 11583816001) or anti-Myc antibodies (Roche, cat. no. 1166720300 ) as a control, overnight at $4{ }^{\circ} \mathrm{C}$. The protein $\mathrm{G}$ agarose beads were added to the reaction and the immune complexes were precipitated by incubating for $1 \mathrm{~h}$ at room temperature. The agarose beads were washed once in
PBS supplemented with $1 \mathrm{mM} \mathrm{CaCl}_{2}$ and $1 \mathrm{mM} \mathrm{MgCl}_{2}$, and then boiled in $2 \times \mathrm{SDS}$ loading buffer before immunoblotting.

Immunoblotting. Total protein $(50-100 \mu \mathrm{g})$ in SDS protein buffer $(100 \mathrm{mM}$ Tris- $\mathrm{HCl}$ (pH 6.8), $200 \mathrm{mM}$ dithiothreitol, 4\% SDS, $0.2 \%$ bromophenol blue, $20 \%$ glycerol) was resolved by SDS-PAGE and transferred to polyvinylidine difluoride membrane. Membranes were blocked in 5\% skim milk-TBST (tris-buffered saline in $0.05 \%$ Tween 20 ) and incubated with a 1 in 1,000 dilution of the following primary antibodies: rabbit anti-H3 (Cell Signalling Technology, Danvers, MA, USA), rabbit anti-H3K27me3 (Millipore), mouse anti-EcR-B1 (AD4.4, Developmental Studies Hybridoma Bank) and anti- $\alpha$ tubulin (Abcam). Secondary antibodies were anti-rabbit AP (Millipore) and anti-mouse AP (Millipore) used at 1 in 2000 dilution. Proteins were detected by enhanced chemifluorescence or enhanced chemiluminescence (ECL-Plus, Amersham/Pharmacia, Piscataway, NJ, USA). Original immunoblots are shown in Supplementary Figure S6.

Fly stocks. $d U T X^{1}, d U T X^{2}$ and $U A S-d U T X, U A S-d U T X^{j m j C}$ are described ${ }^{16}$. The following stocks were from the Bloomington stock centre (Bloomington, IN, USA): $w^{1118}$, hsFLP; $u b i$-GFP FRT40A, Sgs3-GAL4, UAS-EcR-RNAi 104. All flies were maintained and crosses performed at $25^{\circ} \mathrm{C}$.

Measurement of ecdysone titre. Pupae (six pupae per set) were collected for both control and $d U T X^{1}$ mutant at $12 \mathrm{~h} \mathrm{RPF}$, snap-frozen in liquid nitrogen and stored at $-80^{\circ} \mathrm{C}$. The pupae were weighed before extracting ecdysteroid. Samples were homogenized in $300 \mu \mathrm{l}$ of methanol and centrifuged at $16,000 \mathrm{~g}$ for $20 \mathrm{~min}$ at $4{ }^{\circ} \mathrm{C}$. This was repeated twice and the combined methanol supernatants were dried before resuspending in enzyme immunoassay (EIA) buffer (Cayman Chemicals, Inc., USA). Ecdysteroid levels were measured as 20E (Sigma-Aldrich, USA) equivalents. The assay was performed using 20-hydroxyecdysone antiserum, 20hydroxyecdysone AChE Tracer and Ellmann reagent (all from Cayman Chemicals, Inc., USA). Briefly, $50 \mu \mathrm{l}$ of the standards and diluted samples were incubated with $50 \mu \mathrm{l}$ of tracer and anti-serum in anti-rabbit IgG coated ELISA plates overnight, washed five times and developed by adding $200 \mu \mathrm{l}$ Ellman's reagent. The absorbance was read at $405 \mathrm{~nm}$ and all assays were performed in triplicate.

Histology. For hematoxylin and eosin staining of whole pupal sections, $14 \mathrm{~h}$ and $24 \mathrm{~h}$ RPF pupae were fixed in FAAG (85\% ethanol, $4 \%$ formaldehyde, $5 \%$ acetic acid and $1 \%$ glutaraldehyde), then paraffin-embedded prior to sectioning and then hematoxylin and eosin-stained ${ }^{27}$. The controls were $w^{1118}$ Drosophila alone and crossed to Sgs3-GAL4. For each genotype a minimum of 10 pupae were examined using a stereozoom microscope (Olympus, Tokyo, Japan).

Immunohistochemistry. Salivary glands of the required genotype were dissected from appropriately staged animals in PBS then fixed with $4 \%$ paraformaldehyde in PBS for $20 \mathrm{~min}$ at room temperature. Primary antibodies were rabbit anti-cleaved caspase-3 (Cell signalling, \#9661), rabbit anti-H3K27me3 (Millipore) and antiLamin Dm0 (DSHB, ADL67.10) used at 1 in 100 dilution. Secondary antibodies were anti-rabbit Alexa-FLUOR 488, anti-rabbit Alexa-FLUOR 568 and anti-mouse Alexa-FLUOR 568 (Molecular Probes, Eugene, CA, USA) used at 1 in 200 dilution. Hoechst 33342 (Sigma-Aldrich) was used to stain DNA. The samples were imaged with a Biorad confocal microscope (Bio-Rad Microscience, UK; at Detmold Imaging Core Facility, Hanson Institute, Adelaide, SA, Australia).

Polytene chromosome immunostaining. Salivary glands from third instar larvae were dissected and fixed on coverslips in $4 \%$ paraformaldehyde in $45 \%$ glacial acetic acid for 5-10 min. The coverslips were placed on a microscope slide and then the chromosomes were spread using a blunt pair of forceps. The slides were then submerged in liquid nitrogen before removing the coverslip. The samples were blocked in $0.5 \%$ Bovine Serum Albumin 0.1\% Triton-X in PBS (PBT) for $1 \mathrm{~h}$ prior to incubation with 1 in 100 dilution of anti-EcR-B1 and anti-dUTX ${ }^{23}$ overnight at $4{ }^{\circ} \mathrm{C}$. Secondary antibodies were anti-rabbit Alexa-FLUOR 488, and anti-mouse Alexa-FLUOR 568 (Molecular Probes, Eugene, CA, USA) used at 1 in 200 dilution. Hoechst 33342 (Sigma-Aldrich) was used to stain DNA. The samples were imaged by confocal microscopy (Detmold Imaging Core Facility, Hanson Institute, Adelaide, SA, Australia).

Live mCherry imaging. To assay for mCherry-Atg8a, salivary glands were dis sected in PBS with Hoechst 33342 (Sigma-Aldrich) to stain DNA and imaged immediately. Images were quantificated using the mean value from histogram function in Photoshop per cell, which represents the average intensity value.

Imaging. Confocal images were obtained using a BioRad Radiance 2100 confocal microscope with Argon ion $488 \mathrm{~nm}(14 \mathrm{mw})$ and Green HeNe $543 \mathrm{~nm}(1.5 \mathrm{mw})$ lasers and an Olympus IX70 inverted microscope with a $\times 40$ UPLAPO (NA $=1.2$ water) objective. The dual labelled samples were imaged with two separate channels (PMT tubes) in a sequential setting. Green fluorescence was excited with an Ar 
$488 \mathrm{~nm}$ laser line and the emission viewed through a HQ515/30 nm narrow band barrier filter in PMT1. Red fluorescence was excited with a HeNe $543 \mathrm{~nm}$ laser line and the emission viewed through a long-pass barrier filter (E570LP) in PMT2. Automatically all signals from PMTs 1 and 2 were merged. Images were captured using Confocal Assistant software for Microsoft Windows (Todd Clark Brelje, Bethesda, MD, USA) and compiled using Photoshop CS5 (Adobe).

Statistical analysis of data. Student's $t$-test, two-tailed equal variance, was used for all statistical analysis unless otherwise stated. Data are expressed as mean \pm s.d. or mean \pm s.e.m., as appropriate. $P<0.05$ was considered significant. $\chi^{2}$ analysis was performed using an online calculation chi-square tool.

\section{References}

1. King-Jones, K. \& Thummel, C. S. Nuclear receptors-a perspective from Drosophila. Nat. Rev. Genet. 6, 311-323 (2005).

2. Lonard, D. M. \& O'Malley, B. W. Nuclear receptor coregulators: judges, juries, and executioners of cellular regulation. Mol. Cell 27, 691-700 (2007).

3. Kumar, S. \& Cakouros, D. Transcriptional control of the core cell-death machinery. Trends Biochem. Sci. 29, 193-199 (2004).

4. Latham, J. A. \& Dent, S. Y. Cross-regulation of histone modifications. Nat. Struct. Mol. Biol. 14, 1017-1024 (2007).

5. Rando, O. J. Global patterns of histone modifications. Curr. Opin. Genet. Dev. 17, 94-99 (2007).

6. Schuettengruber, B., Chourrout, D., Vervoort, M., Leblanc, B. \& Cavalli, G. Genome regulation by polycomb and trithorax proteins. Cell 128, 735-745 (2007).

7. Sedkov, Y. et al. Methylation at lysine 4 of histone H3 in ecdysone-dependent development of Drosophila. Nature 426, 78-83 (2003).

8. Stratmann, A. \& Haendler, B. Histone demethylation and steroid receptor function in cancer. Mol. Cell Endocrinol. 348, 12-20 (2012).

9. Agger, K. et al. UTX and JMJD3 are histone H3K27 demethylases involved in HOX gene regulation and development. Nature 449, 731-734 (2007).

10. De Santa, F. et al. The histone H3 lysine-27 demethylase Jmjd3 links inflammation to inhibition of polycomb-mediated gene silencing. Cell 130, 1083-1094 (2007).

11. Hong, S. et al. Identification of JmjC domain-containing UTX and JMJD3 as histone H3 lysine 27 demethylases. Proc. Natl Acad. Sci. USA 104, 18439-18444 (2007).

12. Jepsen, K. et al. SMRT-mediated repression of an $\mathrm{H} 3 \mathrm{~K} 27$ demethylase in progression from neural stem cell to neuron. Nature 450, 415-419 (2007).

13. Lan, F. et al. A histone $\mathrm{H} 3$ lysine 27 demethylase regulates animal posterior development. Nature 449, 689-694 (2007).

14. Lee, M. G. et al. Demethylation of H3K27 regulates polycomb recruitment and H2A ubiquitination. Science 318, 447-450 (2007).

15. Swigut, T. \& Wysocka, J. H3K27 demethylases, at long last. Cell 131, 29-32 (2007).

16. Seenundun, S. et al. UTX mediates demethylation of H3K27me3 at musclespecific genes during myogenesis. EMBO J. 29, 1401-1411 (2010).

17. Lee, S., Lee, J. W. \& Lee, S. K. UTX, a histone H3-lysine 27 demethylase, acts as a critical switch to activate the cardiac developmental program. Dev. Cell 22, 25-37 (2012).

18. Mansour, A. A. et al. The H3K27 demethylase Utx regulates somatic and germ cell epigenetic reprogramming. Nature 488, 409-413 (2012).

19. Shpargel, K. B., Sengoku, T., Yokoyama, S. \& Magnuson, T. UTX and UTY demonstrate histone demethylase-independent function in mouse embryonic development. PLoS Genet. 8, e1002964 (2012).

20. Wang, C. et al. UTX regulates mesoderm differentiation of embryonic stem cells independent of H3K27 demethylase activity. Proc. Natl Acad. Sci. USA 109, 15324-15329 (2012).

21. Welstead, G. G. et al. X-linked H3K27me3 demethylase Utx is required for embryonic development in a sex-specific manner. Proc. Natl Acad. Sci. USA 109, 13004-13009 (2012).

22. Miller, S. A., Mohn, S. E. \& Weinmann, A. S. Jmjd3 and UTX play a demethylase-independent role in chromatin remodeling to regulate T-box family member-dependent gene expression. Mol. Cell 40, 594-605 (2010).

23. Herz, H. M. et al. The H3K27me3 demethylase dUTX is a suppressor of Notchand Rb-dependent tumors in Drosophila. Mol. Cell Biol. 30, 2485-2497 (2010).

24. Lee, C. Y., Simon, C. R., Woodard, C. T. \& Baehrecke, E. H. Genetic mechanism for the stage- and tissue-specific regulation of steroid triggered programmed cell death in Drosophila. Dev Biol. 252, 138-148 (2002).

25. Lee, C. Y. \& Baehrecke, E. H. Steroid regulation of autophagic programmed cell death during development. Development 128, 1443-1455 (2001).

26. Berry, D. L. \& Baehrecke, E. H. Growth arrest and autophagy are required for salivary gland cell degradation in Drosophila. Cell 131, 1137-1148 (2007).

27. Daish, T. J., Mills, K. \& Kumar, S. Drosophila caspase DRONC is required for specific developmental cell death pathways and stress-induced apoptosis. Dev. Cell 7, 909-915 (2004).
28. Mills, K. et al. The Drosophila melanogaster Apaf-1 homologue ARK is required for most, but not all, programmed cell death. J. Cell. Biol. 172, 809-815 (2006).

29. Gorski, S. M. et al. A SAGE approach to discovery of genes involved in autophagic cell death. Curr Biol. 13, 358-363 (2003).

30. Lee, C. Y. et al. Genome-wide analyses of steroid- and radiation-triggered programmed cell death in Drosophila. Curr Biol. 13, 350-357 (2003).

31. Jiang, C., Lamblin, A. F., Steller, H. \& Thummel, C. S. A steroid-triggered transcriptional hierarchy controls salivary gland cell death during Drosophila metamorphosis. Mol. Cell 5, 445-455 (2000).

32. Dorstyn, L., Colussi, P. A., Quinn, L. M., Richardson, H. \& Kumar, S. DRONC an ecdysone-inducible Drosophila caspase. Proc. Natl Acad. Sci. USA 96, 43074312 (1999).

33. Cakouros, D., Daish, T., Martin, D., Baehrecke, E. H. \& Kumar, S. Ecdysoneinduced expression of the caspase DRONC during hormone-dependent programmed cell death in Drosophila is regulated by Broad-Complex. J. Cell Biol. 157, 985-995 (2002).

34. Daish, T. J., Cakouros, D. \& Kumar, S. Distinct promoter regions regulate spatial and temporal expression of the Drosophila caspase dronc. Cell Death Differ. 10, 1348-1356 (2003).

35. Cakouros, D., Daish, T. J., Mills, K. \& Kumar, S. An arginine-histone methyltransferase, CARMER, coordinates ecdysone-mediated apoptosis in Drosophila cells. J. Biol. Chem. 279, 18467-18471 (2004).

36. Cakouros, D. et al. dLKR/SDH regulates hormone-mediated histone arginine methylation and transcription of cell death genes. J. Cell. Biol. 182, 481-495 (2008).

37. Ress, C., Holtmann, M., Maas, U., Sofsky, J. \& Dorn, A. 20-Hydroxyecdysoneinduced differentiation and apoptosis in the Drosophila cell line, $1(2) \mathrm{mbn}$. Tissue Cell 32, 464-477 (2000).

38. Kilpatrick, Z. E., Cakouros, D. \& Kumar, S. Ecdysone-mediated up-regulation of the effector caspase DRICE is required for hormone-dependent apoptosis in Drosophila cells. J. Biol. Chem. 280, 11981-11986 (2005).

39. Heery, D. M., Kalkhoven, E., Hoare, S. \& Parker, M. G. A signature motif in transcriptional co-activators mediates binding to nuclear receptors. Nature $\mathbf{3 8 7}$, 733-736 (1997)

40. Denton, D. et al. Relationship between growth arrest and autophagy in midgut programmed cell death in Drosophila. Cell Death Differ. 19, 1299-1307 (2012).

41. Baehrecke, E. H. \& Thummel, C. S. The Drosophila E93 gene from the 93F early puff displays stage- and tissue-specific regulation by 20-hydroxyecdysone. Dev Biol. 171, 85-97 (1995).

42. Burtis, K. C., Thummel, C. S., Jones, C. W., Karim, F. D. \& Hogness, D. S. The Drosophila 74EF early puff contains E74, a complex ecdysone-inducible gene that encodes two ets-related proteins. Cell 61, 85-99 (1990).

43. DiBello, P. R., Withers, D. A., Bayer, C. A., Fristrom, J. W. \& Guild, G. M. The Drosophila Broad-Complex encodes a family of related proteins containing zinc fingers. Genetics 129, 385-397 (1991)

44. Thummel, C. S. Flies on steroids-Drosophila metamorphosis and the mechanisms of steroid hormone action. Trends Genet. 12, 306-310 (1996).

45. Jiang, C., Baehrecke, E. H. \& Thummel, C. S. Steroid regulated programmed cell death during Drosophila metamorphosis. Development 124, 4673-4683 (1997).

46. Lee, C. Y., Wendel, D. P., Reid, P., Lam, G., Thummel, C. S. \& Baehrecke, E. H. E93 directs steroid-triggered programmed cell death in Drosophila. Mol. Cell 6, 433-443 (2000).

47. Grasso, C. S. et al. The mutational landscape of lethal castration-resistant prostate cancer. Nature 487, 239-243 (2012).

48. Settembre, C. et al. TFEB links autophagy to lysosomal biogenesis. Science 332, 1429-1433 (2011).

49. Füllgrabe, J. et al. The histone H4 lysine 16 acetyltransferase hMOF regulates the outcome of autophagy. Nature 200, 468-471 (2013).

50. Denton, D., Nicolson, S. \& Kumar, S. Cell death by autophagy: facts and apparent artefacts. Cell Death Differ. 19, 87-95 (2012).

51. Dorstyn, L., Mills, K., Lazebnik, Y. \& Kumar, S. The two cytochrome $c$ species, DC3 and DC4, are not required for caspase activation and apoptosis in Drosophila cells. J. Cell Biol. 167, 405-410 (2004).

52. Denton, D., Mills, K. \& Kumar, S. Methods and protocols for studying cell death in Drosophila. Methods Enzymol. 446, 17-37 (2008).

53. Yao, T. P., Segraves, W. A., Oro, A. E., McKeown, M. \& Evans, R. M. Drosophila ultraspiracle modulates ecdysone receptor function via heterodimer formation. Cell 71, 63-72 (1992).

\section{Acknowledgements}

This work was supported by the National Health and Medical Research Counci (NHMRC) Project Grants 626903 and 626923, and a Senior Principal Research Fellowship 1002863 to S.K., as well as R01 GM068016 from the National Institutes of Health (NIH) to A.B. We thank the Australian Drosophila Research Support Facility, Vienna Drosophila RNAi Center and Bloomington Drosophila Stock Center for Drosophila stocks, Developmental Studies Hybridoma Bank (University of Iowa) for antibodies, 
Earanee Niedzwiecki for maintaining Drosophila stocks, Amanda Rogers for tissue processing and Leonie Quinn for advice on polytene chromosome preparation and staining.

\section{Author contributions}

S.K., D.C. and D.D. conceptualized and designed the project; D.D., K.M., M.T.A.-H., S.N., W.Z., N.L. and D.C. designed and performed experimental work; D.D., S.K., M.A.H., N.L. and S.N. analysed the data; A.B. contributed the reagents and advice; D.D., M.A.-H. and S.K. prepared the manuscript. M.A.-H. and N.L. contributed equally to this manuscript and thus should be considered as equal second authors.

\section{Additional information}

Supplementary Information accompanies this paper at http://www.nature.com/ naturecommunications

Competing financial interests: The authors declare no competing financial interests.

Reprints and permission information is available online at http://npg.nature.com/ reprintsandpermissions/

How to cite this article: Denton, D. et al. UTX coordinates steroid hormonemediated autophagy and cell death. Nat. Commun. 4:2916 doi: 10.1038/ncomms3916 (2013). 